\title{
Remarks on the asymptotically discretely self-similar solutions of the Navier-Stokes and the Euler equations
}

\author{
Dongho Chae \\ Chung-Ang University \\ Department of Mathematics \\ Seoul 156-756, Republic of Korea \\ e-mail: dchae@cau.ac.kr
}

\begin{abstract}
We study scenarios of self-similar type blow-up for the incompressible NavierStokes and the Euler equations. The previous notions of the discretely (backward) self-similar solution and the asymptotically self-similar solution are generalized to the locally asymptotically discretely self-similar solution. We prove that there exists no such locally asymptotically discretely self-similar blow-up for the 3D Navier-Stokes equations if the blow-up profile is a time periodic function belonging to $C^{1}\left(\mathbb{R} ; L^{3}\left(\mathbb{R}^{3}\right) \cap C^{2}\left(\mathbb{R}^{3}\right)\right)$. For the $3 \mathrm{D}$ Euler equations we show that the scenario of asymptotically discretely self-similar blow-up is excluded if the blow-up profile satisfies suitable integrability conditions.
\end{abstract}

Mathematics Subject Classification(2000): 76B03, 35Q31

\section{The main theorems}

\section{$1.1 \quad$ Navier-Stokes equations}

We consider the Cauchy problem of the 3D Navier-stokes equations.

$$
(N S)\left\{\begin{array}{l}
\partial_{t} v+v \cdot \nabla v=-\nabla p+\Delta v \\
\operatorname{div} v=0 \\
v(x, 0)=v_{0}(x)
\end{array}\right.
$$

where $v(x, t)=\left(v_{1}(x, t), v_{2}(x, t), v_{3}(x, t)\right)$ is the velocity, $p=p(x, t)$ is the pressure, and $v_{0}(x)$ is the initial data satisfying $\operatorname{div} v_{0}=0$. We study the possibility of finite time blow-up of smooth solution of (NS). By translation of time we may assume that the solution is smooth for $t<0$, and the blow-up happens at $t=0$. We say a solution 
$v(x, t)$ to $(\mathrm{NS})$ is a (backward) self-similar blowing up solution at $t=0$ if there exists $(V, P)$ such that

$$
v(x, t)=\frac{1}{\sqrt{-t}} V\left(\frac{x}{\sqrt{-t}}\right), \quad p(x, t)=-\frac{1}{t} P\left(\frac{x}{\sqrt{-t}}\right),
$$

For such solution we have $\lambda v\left(\lambda x, \lambda^{2} t\right)=v(x, t)$ for all $\lambda \in \mathbb{R}$ and for all $(x, t) \in$ $\mathbb{R}^{3} \times(-\infty, 0)$, The nonexistence of nontrivial solution given by (1.6) was shown by Nečas-Råžička-Šverák([15]), and Tsai([20])(see also [14]). It can also be deduced from the result of [8]. These results are generalized by introduction of the more general notion of the asymptotically self-similar blow-up solutions, and their exclusion in [2], which was motivated by earlier study of asymptotically self-similar solutions for the nonlinear heat equation by Giga and Kohn[9, 10](see also [12]). A different direction of generalization of the notion can be done by considering the discretely (backward) self-similar blow-up at $(x, t)=(0,0)$ for a solution of (NS) is a solution $v(x, t)$, which means that there exists $\lambda \neq 1$ such that $\lambda v\left(\lambda x, \lambda^{2} t\right)=v(x, t)$ for all $(x, t) \in \mathbb{R}^{3} \times(-\infty, 0)$. We note that the notion of forward discretely self-similar solutions to (NS) was studied earlier by Tsai in [19](see also [7]). If we make a self-similar transform, $(v, p) \rightarrow(V, P) \in\left[C^{\infty}\left(\mathbb{R}^{3+1}\right)\right]^{2}$ by the formula,

$$
v(x, t)=\frac{1}{\sqrt{-t}} V(y, s), \quad p(x, t)=-\frac{1}{t} P(y, s),
$$

where $y=x / \sqrt{-t}$ and $s=-\log (-t)$, then, $(V, P)$ satisfies

$$
\left\{\begin{array}{l}
V_{s}+\frac{1}{2} V+\frac{1}{2}(y \cdot \nabla) V+(V \cdot \nabla) V=-\nabla P+\Delta V, \\
\operatorname{div} V=0
\end{array}\right.
$$

which has the periodicity in time, $V(y, s)=V\left(y, s+S_{0}\right)$ for all $(y, s) \in \mathbb{R}^{3+1}$ with $S_{0}=-2 \log \lambda$. We first prove the nonexistence of discretely self-similar blow-up for the $L^{3}\left(\mathbb{R}^{3}\right)$ solution to (NS), which is an easy consequence of [18], as is shown in the next section.

Theorem 1.1. If $v \in C\left(-\infty, 0 ; L^{3}\left(\mathbb{R}^{3}\right)\right)$ a solution to (NS), which blows up at $t=0$, then $t=0$ is not a time for discretely self-similar blow up.

Next we consider more general possibility of locally asymptotically discretely selfsimilar blow-up. Let $q \in[1, \infty)$, and $v \in C\left(-\infty, 0 ; L_{l o c}^{q}\left(\mathbb{R}^{3}\right)\right)$ be a solution of $(\mathrm{NS})$, which blows up at $t=0$. We say that $(x, t)=(0,0)$ is a space-time point of locally asymptotically discretely self-similar blow-up in the sense of $L^{q}$ if there exist $R>0$ and a solenoidal vector field $\bar{V}(y, s) \in C\left(\mathbb{R} ; L^{q}\left(\mathbb{R}^{3}\right)\right)$ with $\bar{V}(y, s)=\bar{V}\left(y, s+S_{0}\right)$ for some $S_{0} \neq 0$ and for all $(y, s) \in \mathbb{R}^{3} \times \mathbb{R}$ such that

$$
\lim _{t \uparrow 0}(-t)^{\frac{q-3}{2 q}} \sup _{t<\tau<0}\left\|v(\cdot, \tau)-\frac{1}{\sqrt{-\tau}} \bar{V}\left(\frac{\cdot}{\sqrt{-\tau}},-\log (-\tau)\right)\right\|_{L^{q}(B(0, R \sqrt{-t}))}=0 .
$$

Note that this is much more general notion than the discrete self-similarity, which corresponds to the equality inside of the norm in (1.4) for all $R>0$. The following is a generalization of Theorem 1.2 of [2] and Theorem 1.1 above. 
Theorem 1.2. Let $v \in C\left(-\infty, 0 ; L_{\text {loc }}^{3}\left(\mathbb{R}^{3}\right)\right)$ be a solution to $(N S)$, which blows up at $t=0$, then it is not a time for locally asymptotically discretely self-similar blow-up in the sense of $L^{q}\left(\mathbb{R}^{3}\right)$ if $q \in[2, \infty]$, and the blow-up profile satisfies $\bar{V} \in C\left(\mathbb{R} ; L^{3}\left(\mathbb{R}^{3}\right) \cap\right.$ $\left.C^{2}\left(\mathbb{R}^{3}\right)\right)$.

Remark 1.1 Although we considered possible blow-up at $(x, t)=(0,0)$ in the above, the result holds also for any $(x, t)$ by translation.

\subsection{The Euler equations}

The question of the finite time blow-up for the 3D Euler equations is also one of the most outstanding problem in the mathematical fluid mechanics(see [13] for the physical and mathematical backgrounds on the problem and [1, 6] for well-known partial results in this direction). Here we study the possibility of existence of selfsimilar type blow-up for a solution to the 3D Euler equations.

$$
(E)\left\{\begin{array}{l}
\partial_{t} v+v \cdot \nabla v=-\nabla p, \\
\operatorname{div} v=0 \\
v(x, 0)=v_{0}(x)
\end{array}\right.
$$

We say a solution $v$ is a self-similar blowing up solution at $t=0$ if there exists $(V, P)$ such that

$$
v(x, t)=\frac{1}{(-t)^{\frac{\alpha}{\alpha+1}}} V\left(\frac{x}{(-t)^{\frac{1}{\alpha+1}}}\right), \quad p(x, t)=\frac{1}{(-t)^{\frac{2 \alpha}{\alpha+1}}} P\left(\frac{x}{(-t)^{\frac{1}{\alpha+1}}}\right) .
$$

For physical motivation on this type scenarios we refer [16]. For such $(v, p)$ we have the following scaling invariance $v(x, t)=\lambda^{\alpha} v\left(\lambda x, \lambda^{\alpha+1} t\right)$ and $p(x, t)=\lambda^{2 \alpha} v\left(\lambda x, \lambda^{\alpha+1} t\right)$ for all $\lambda>0, \alpha \in \mathbb{R}$, and for all $(x, t) \in \mathbb{R}^{3} \times(-\infty, 0)$. The nonexistence of nontrivial selfsimilar blowing up solution under suitable assumption on the blow-up profile $V$ was obtained in [4]. A discretely self-similar solution $v$ is a solenoidal vector field, for which there exist $\lambda>0, \alpha>-1$ such that $v(x, t)=\lambda^{\alpha} v\left(\lambda x, \lambda^{\alpha+1} t\right) \quad \forall(x, t) \in \mathbb{R}^{3} \times(-\infty, 0)$. If we represent $(v, p)$ by

$$
v(x, t)=\frac{1}{(-t)^{\frac{\alpha}{\alpha+1}}} V(y, s), \quad p(x, t)=\frac{1}{(-t)^{\frac{2 \alpha}{\alpha+1}}} P(y, s),
$$

where $y=x /(-t)^{\frac{1}{\alpha+1}}$ and $s=-\log (-t)$. then the discrete self-similarity of the solution $(v, p)$ is equivalent to that $(V, P)$ is a solution to

$$
\left\{\begin{array}{l}
V_{s}+\frac{\alpha}{\alpha+1} V+\frac{1}{\alpha+1}(y \cdot \nabla) V+(V \cdot \nabla) V=-\nabla P, \\
\operatorname{div} V=0
\end{array}\right.
$$

which satisfies $V(y, s)=V\left(y, s+S_{0}\right)$ for all $(y, s) \in \mathbb{R}^{3+1}$ with $S_{0}=-(\alpha+1) \log \lambda$. The following result on the nonexistence of nontrivial discretely self-similar blow-up for the 3D Euler equations is proved in [5]. 
Theorem 1.3. Let $V(y, s) \in C^{1}\left(\mathbb{R}^{3+1}\right)$ be a time periodic solution of (1.7) with period $S_{0}>0$.

(i) Let $V$ satisfies either one of the following conditions:

(a) $V(y, s) \in C_{y}^{2} C_{s}^{1}\left(\mathbb{R}^{3+1}\right)$, and

$$
\Omega:=\operatorname{curl} V \in \cup_{r>0} \cap_{0<q<r} L^{q}\left(\mathbb{R}^{3} \times\left[0, S_{0}\right]\right) .
$$

(b) $\lim _{|y| \rightarrow \infty} \sup _{0<s<S_{0}}|\nabla V(y, s)|=0$, and $\Omega \in L^{q}\left(\mathbb{R}^{3} \times\left[0, S_{0}\right]\right)$ for some $q \in$ $\left(0, \frac{3}{1+\alpha}\right)$.

Then, $\Omega=0$. If we further assume $\lim _{|y| \rightarrow \infty} V(y, s)=0 \forall s \in\left[0, S_{0}\right)$, then $V=0$.

(ii) Let $-1<\alpha \leq 3 / p$ or $3 / 2<\alpha<\infty$, and $V \in L^{p}\left(\mathbb{R}^{3} \times\left(\left[0, S_{0}\right]\right)\right.$ for some $3 \leq p \leq \infty$. We also assume that the pressure is given by $-\Delta_{y} P(\cdot, s)=$ $\sum_{i, j} \partial_{i} \partial_{j}\left(V_{i} V_{j}(\cdot, s)\right)$. Then, $V=0$.

In the next section we will prove following theorem, which is an extension of the part (i) (b) of the above theorem.

Theorem 1.4. Let $V(y, s) \in C^{1}\left(\mathbb{R}^{3+1}\right)$ be a time periodic solution of (1.7) with period $S_{0}>0$ satisfying

$$
\lim _{|y| \rightarrow \infty} \sup _{0<s<S_{0}}|\nabla V(y, s)|=0
$$

and

$$
\int_{0}^{S_{0}} \int_{\mathbb{R}^{3}}|\Omega(y, s)|^{q}(1+|y|)^{\eta} d y d s<\infty,
$$

where $q \in\left(0, \frac{3+\eta}{1+\alpha}\right)$ with $\alpha>-1, \eta>-3$. Then, $\Omega=0$. Therefore if we further assume $\lim _{|y| \rightarrow \infty} V(y, s)=0 \forall s \in\left[0, S_{0}\right)$, then $V=0$.

Note that case of $\eta=0$ of the above theorem corresponds to the part (i)(b) of Theorem 1.3. We now introduce more general notion of the asymptotically discretely self-similar blow-up for the 3D incompressible Euler equations. Let $v \in C^{1}\left(\mathbb{R}^{3+1}\right)$ be a solution to $(\mathrm{E})$, which blows up at $t=0$. We say that $t=0$ is a time for asymptotically discretely self-similar blow-up if there exist $\alpha>-1$ and a solenoidal vector field $\bar{V} \in C^{1}\left(\mathbb{R}^{3+1}\right)$, which is a time periodic solution to (1.7), and satisfies the following convergence conditions:

$$
\lim _{t \uparrow 0}(-t)\left\|\nabla v(\cdot, t)+\frac{1}{t} \nabla \bar{V}\left(\frac{\cdot}{(-t)^{\frac{1}{\alpha+1}}},-\log (-t)\right)\right\|_{L^{\infty}}=0,
$$

and there exists $\varepsilon_{0}>0$ such that

$$
\sup _{-\varepsilon_{0}<t<0}(-t)^{\frac{3-\alpha}{\alpha+1}}\left\|v(\cdot, t)-\frac{1}{(-t)^{\frac{\alpha}{\alpha+1}}} \bar{V}\left(\frac{\cdot}{(-t)^{\frac{1}{\alpha+1}}},-\log (-t)\right)\right\|_{L^{1}}<\infty .
$$

The following is a generalization of the corresponding results in [2, 3]. 
Theorem 1.5. Let $T>0$ and $v \in C\left([-T, 0) ; H^{m}\left(\mathbb{R}^{3}\right)\right), m>5 / 2$, be a classical solution to $(E)$, which blows up at $t=0$. Then, $t=0$ is not a time for asymptotically discretely self-similar blow-up if the blow-up profile $\bar{V} \in C^{1}\left(\mathbb{R}^{3+1}\right)$ satisfies either the conditions (i) (a) or (i)(b) of Theorem 1.3, or the conditions (1.8)-(1.9) of Theorem 1.4, together with the condition $\lim _{|y| \rightarrow \infty} \sup _{s \in \mathbb{R}}|V(y, s)|=0$.

\section{Proof of the main theorems}

Proof of Theorem 1.1 We recall the main result in [18], saying that $t=0$ is the blow-up time for the solution $v \in C\left(-\infty, 0 ; L^{3}\left(\mathbb{R}^{3}\right)\right)$ only if

$$
\lim _{t \uparrow 0}\|v(\cdot, t)\|_{L^{3}}=\infty .
$$

By the discrete self-similarity there exists $0<\lambda \neq 1$ such that

$$
\lambda^{k} v\left(\lambda^{k} x, \lambda^{2 k} t\right)=v(x, t) \quad \forall k \in \mathbb{Z}
$$

Hence,

$$
\left\|v\left(\cdot, \lambda^{2 k} t\right)\right\|_{L^{3}}=\|v(\cdot, t)\|_{L^{3}} \quad \forall t \in(-\infty, 0) .
$$

Passing $k \rightarrow \infty$ if $\lambda \in(0,1)$, while passing $k \rightarrow-\infty$ if $\lambda \in(1, \infty)$, one has for $t_{k}=\lambda^{k} t$

$$
\|v(\cdot, t)\|_{L^{3}}=\lim _{t_{k} \rightarrow 0}\left\|v\left(\cdot, t_{k}\right)\right\|_{L^{3}}=\infty \quad \forall t \in(-\infty, 0),
$$

which is contradiction to the fact that $v \in C\left(-\infty, 0 ; L^{3}\left(\mathbb{R}^{3}\right)\right)$.

For the proof of Theorem 1.2 we shall use the following result, which is Theorem 1.1 of [1].

Lemma 2.1. Let $q \in[3 / 2, \infty]$. Suppose $v$ is a suitable weak solution of $(N S)$ in a cylinder, say $Q=B\left(0, r_{1}\right) \times\left(-r_{1}^{2}, 0\right)$ for some $r_{1}>0$. Then, there exists a constant $\eta=\eta(q)>0$ such that if

$$
\lim \sup _{r \downarrow 0}\left\{r^{\frac{q-3}{q}} \text { ess } \sup _{\substack{-r^{2}<t<0\\}}\|v(\cdot, t)\|_{L^{q}(B(0, r))}\right\} \leq \eta,
$$

then $v$ is Hölder continuous both in space and time variables near $(0,0) \in \mathbb{R}^{3+1}$.

Proof of Theorem 1.2 We use the self-similar variables $y=x / \sqrt{-t}, \quad s=$ $-\log (-t)$, and transform $(v, p) \rightarrow(V, P)$ as previously,

$$
v(x, t)=\frac{1}{\sqrt{-t}} V\left(\frac{x}{\sqrt{-t}},-\log (-t)\right), \quad p(x, t)=-\frac{1}{t} P\left(\frac{x}{\sqrt{-t}},-\log (-t)\right) .
$$

Then, substituting $(v, p)$ into (NS), we find that $(V, P)$ satisfies (1.3). We observe that the condition (1.4) for some $R \in(0, \infty)$ is equivalent to

$$
\lim _{t \uparrow 0}(-t)^{\frac{q-3}{2 q}} \sup _{t<\tau<0}\left\|v(\cdot, \tau)-\frac{1}{\sqrt{-\tau}} \bar{V}\left(\frac{\cdot}{\sqrt{-\tau}},-\log (-\tau)\right)\right\|_{L^{q}(B(0, R \sqrt{-t}))}=0
$$


for all $R \in(0, \infty)$ (see e.g. the argument in the proof of Theorem 1.5, pp. 446, [2]), which implies, in terms of $V$, that

$$
\lim _{s \rightarrow \infty}\|V(\cdot, s)-\bar{V}(\cdot, s)\|_{L^{q}(B(0, R))}=0
$$

for all $R \in(0, \infty)$. We now show that this implies that $\bar{V} \in C_{t}^{1} C_{x}^{2}\left(\mathbb{R}^{3+1}\right)$ is a solution to (1.3). Let $\xi \in C_{0}^{1}\left(0, S_{0}\right)$ and $\phi=\left(\phi_{1}, \phi_{2}, \phi_{3}\right) \in C_{0}^{2}\left(\mathbb{R}^{3}\right)$ with $\operatorname{div} \phi=0$. We multiply the first equations of (1.3) by $\xi\left(s-S_{0} n\right) \phi(y)$, and integrate it over $\mathbb{R}^{3} \times\left[n, n+S_{0}\right]$, and then we integrate by parts to obtain:

$$
\begin{aligned}
- & \int_{0}^{S_{0}} \int_{\mathbb{R}^{3}} \xi_{s}(s) \phi(y) \cdot V\left(y, s+S_{0} n\right) d y d s-\int_{0}^{S_{0}} \int_{\mathbb{R}^{3}} \xi(s) V\left(y, s+S_{0} n\right) \cdot \phi(y) d y d s \\
& -\frac{1}{2} \int_{0}^{S_{0}} \int_{\mathbb{R}^{3}} \xi(s) V\left(y, s+S_{0} n\right) \cdot(y \cdot \nabla) \phi(y) d y d s \\
& -\int_{0}^{S_{0}} \int_{\mathbb{R}^{3}} \xi(s)\left[V\left(y, s+S_{0} n\right) \cdot\left(V\left(y, s+S_{0} n\right) \cdot \nabla\right) \phi(y)\right] d y d s \\
= & \int_{0}^{S_{0}} \int_{\mathbb{R}^{3}} \xi(s) V\left(y, s+S_{0} n\right) \cdot \Delta \phi(y) d y d s
\end{aligned}
$$

Similarly we multiply the second equations of (1.3) by $\xi\left(s-S_{0} n\right) \psi(y)$ with $\psi \in$ $C_{0}^{1}\left(\mathbb{R}^{3}\right)$, and integrate it over $\mathbb{R}^{3} \times\left[n, n+S_{0}\right]$, we have

$$
\int_{0}^{S_{o}} \int_{\mathbb{R}^{3}} V\left(s+S_{0} n\right) \cdot \nabla \psi(y) \xi(s) d y d s=0 .
$$

Passing to the limit $n \rightarrow \infty$ in (2.7)-(2.8), and observing that $V\left(\cdot, s+S_{0} n\right) \rightarrow \bar{V}(\cdot, s)$ in $L_{\text {loc }}^{q}\left(\mathbb{R}^{3}\right) \hookrightarrow L_{\text {loc }}^{2}\left(\mathbb{R}^{3}\right)$ for $q \in[2, \infty]$, we find that $\left.\bar{V} \in C_{s}^{1} C_{y}^{2}\left(\mathbb{R}^{3+1}\right)\right)$ satisfies (2.7) and (2.8). Integrating by part in (2.7) and (2.8) for $\bar{V}$ respectively, we obtain

$$
\int_{\mathbb{R}^{3}} \int_{0}^{S_{0}}\left[\overline{V_{s}}+\frac{1}{2} \bar{V}+\frac{1}{2}(y \cdot \nabla) \bar{V}+(\bar{V} \cdot \nabla) \bar{V}-\Delta \bar{V}\right] \cdot \phi(y) \xi(s) d y d s=0
$$

for all vector test function $\phi \in C_{0}^{2}\left(\mathbb{R}^{3}\right)$ with $\operatorname{div} \phi=0$, and $\xi \in C_{0}^{1}\left(0, S_{0}\right)$, and also for all $\psi \in C^{2}\left(\mathbb{R}^{3}\right)$ we have

$$
\int_{0}^{S_{0}} \int_{\mathbb{R}^{3}}[\operatorname{div} \bar{V}] \psi(y) \xi(s) d y d s=0 .
$$

Therefore there exists $\bar{P} \in C^{1}\left(\mathbb{R}^{3} \times\left[0, S_{0}\right)\right)$ such that

$$
\bar{V}_{s}+\frac{1}{2} \bar{V}+\frac{1}{2}(y \cdot \nabla) \bar{V}+(\bar{V} \cdot \nabla) \bar{V}-\Delta \bar{V}=-\nabla \bar{P}, \quad \operatorname{div} \bar{V}=0 .
$$

Since $\bar{V}$ is a $C\left(\mathbb{R}^{3} ; L^{3}\left(\mathbb{R}^{3}\right) \cap C^{2}\left(\mathbb{R}^{3}\right)\right)$ solution of (1.3) satisfying the periodic condition, $\bar{V}(y, s)=\bar{V}\left(y, s+S_{0}\right)$, we find that $\bar{V}=0$ by Theorem 1.1 . Therefore, the assumption (1.4) is reduced to

$$
\lim _{t \uparrow 0}\left\{(-t)^{\frac{q-3}{2 q}} \sup _{t<\tau<0}\|v(\cdot, \tau)\|_{L^{q}(B(3, R \sqrt{-t}))}\right\}=0
$$


for all $R \in(0, \infty)$. We set $R=1$ and $\sqrt{-t}=r$ in (2.11), then we obtain

$$
\lim _{r \downarrow 0}\left\{r^{\frac{q-3}{q}} \sup _{-r^{2}<\tau<0}\|v(\cdot, \tau)\|_{L^{q}(B(0, r))}\right\}=0 .
$$

Applying Lemma 2.1, we find that the space-time point $(0,0)$ is not a blow-up spacetime point.

In order to prove Theorem 1.3 we recall the following blow-up criterion for the Euler equations, which corresponds to Lemma 2.1 for the Navier-Stokes equations.

Lemma 2.2. Let $m>5 / 2, \varepsilon>0$ and $v \in C\left((-\varepsilon, 0) ; H^{m}\left(\mathbb{R}^{3}\right)\right)$ be a classical solution to (E). Suppose the following inequality holds

$$
\lim \sup _{t \uparrow 0}(-t)\|\nabla v(t)\|_{L^{\infty}}<1
$$

then there exists no blow-up at $t=0$.

For the proof see the proof of Theorem 1.1 in [3] (see also [12] for similar result with different approach).

Proof of Theorem 1.5 We transform the solution $(v, p) \rightarrow(V, P)$ as in (1.6). Then, $(V, P)$ satisfies (1.7). The convergence conditions (1.10)-(1.11) can be written in the self-similar form as

$$
\lim _{s \rightarrow \infty}\|\nabla V(\cdot, s)-\nabla \bar{V}(\cdot, s)\|_{L^{\infty}}=0
$$

and

$$
\sup _{-\log \left(\varepsilon_{0}\right)<s<\infty}\|V(\cdot, s)-\bar{V}(\cdot, s)\|_{L^{1}}<\infty
$$

respectively. From (2.14) and (2.15), using the interpolation for the $L^{p}$ spaces, we have that

$$
\lim _{s \rightarrow \infty}\|V(\cdot, s)-\bar{V}(\cdot, s)\|_{L^{2}\left(B_{R}\right)}=0 \quad \forall R>0,
$$

and repeating the argument of the proof of Theorem 1.2 word by word, we find that $\bar{V}$ is $C^{1}\left(\mathbb{R}^{3} \times\left(0, S_{0}\right)\right)$ solution of (1.7), satisfying the time periodicity and one of the conditions (i) (a) or (i) (b) of Theorem 1.3, or the conditions (1.8)-(1.9) of Theorem 1.4, together with the condition $\lim _{|y| \rightarrow \infty} \sup _{s \in \mathbb{R}}|V(y, s)|=0$. Therefore $\bar{V}=0$, and the condition (1.10) reduces to

$$
\lim _{t \uparrow 0}(-t)\|\nabla v(\cdot, t)\|_{L^{\infty}}=0 .
$$

Thanks Lemma 2.2 we can conclude that $t=0$ is not a blow-up time.

Proof of Theorem 1.4 We consider the vorticity equation of (1.7),

$$
\left\{\begin{array}{l}
\Omega_{s}+\Omega+\frac{1}{\alpha+1}(y \cdot \nabla) \Omega+(V \cdot \nabla) \Omega-(\Omega \cdot \nabla) V=0 \\
\operatorname{div} V=0, \quad \operatorname{curl} V=\Omega
\end{array}\right.
$$


We introduce a cut-off function $\sigma \in C_{0}^{\infty}\left(\mathbb{R}^{N}\right)$ such that

$$
\sigma(|x|)= \begin{cases}1 & \text { if }|x|<1 \\ 0 & \text { if }|x|>2\end{cases}
$$

and $0 \leq \sigma(x) \leq 1$ for $1<|x|<2$. For each $R>0$, we define $\sigma_{R}(x):=\sigma\left(\frac{|x|}{R}\right)$. Given $\rho>0$, we also define a function $\psi=\psi_{\rho}(y)$ as follows.

$$
\psi_{\rho}(y)=\left\{\begin{array}{lr}
1, & \text { if } \quad|y|>\rho+\pi \\
\frac{1}{2} \sin \left(|y|-\rho-\frac{\pi}{2}\right)+\frac{1}{2}, & \text { if } \quad \rho<|y| \leq \rho+\pi \\
0, & \text { if } \quad|y| \leq \rho .
\end{array}\right.
$$

We take $L^{2}\left(\mathbb{R}^{3} \times\left[0, S_{0}\right]\right)$ inner product the first equations of (2.17) by $\Omega|\Omega|^{q-2}|y|^{\eta} \psi_{\rho} \sigma_{R}$, and integration by parts to obtain

$$
\begin{aligned}
0= & \left(1-\frac{3+\eta}{q(\alpha+1)}\right) \int_{0}^{S_{0}} \int_{\mathbb{R}^{3}}|\Omega|^{q}|y|^{\eta} \psi_{\rho} \sigma_{R} d y d s-\frac{1}{\alpha+1} \int_{0}^{S_{0}} \int_{\mathbb{R}^{3}}|\Omega|^{q}|y|^{\eta}(y \cdot \nabla) \psi_{\rho} \sigma_{R} d y d s \\
& -\frac{1}{\alpha+1} \int_{0}^{S_{0}} \int_{\mathbb{R}^{3}}|\Omega|^{q}|y|^{\eta} \psi_{\rho}(y \cdot \nabla) \sigma_{R} d y d s-\frac{\eta}{q} \int_{0}^{S_{0}} \int_{\mathbb{R}^{3}}|\Omega|^{q} V \cdot \frac{y}{|y|}|y|^{\eta-1} \psi_{\rho} \sigma_{R} d y d s \\
& -\frac{1}{q} \int_{0}^{S_{0}} \int_{\mathbb{R}^{3}}|\Omega|^{q}|y|^{\eta}(V \cdot \nabla) \psi_{\rho} \sigma_{R} d y d s-\frac{1}{q} \int_{0}^{S_{0}} \int_{\mathbb{R}^{3}}|\Omega|^{q}|y|^{\eta} \psi_{\rho}(V \cdot \nabla) \sigma_{R} d y d s \\
& \quad-\int_{0}^{S_{0}} \int_{\mathbb{R}^{3}}(\Omega \cdot \nabla) V \cdot \Omega|\Omega|^{q-2}|y|^{\eta} \psi_{\rho} \sigma_{R} d y d s \\
& :=I_{1}+\cdots+I_{7},
\end{aligned}
$$

where the periodicity of $V(y, s)$ and $\Omega(y, s)$ in $s$ was used. We first observe that for all $\eta \in \mathbb{R}$

$$
\int_{0}^{S_{0}} \int_{\mathbb{R}^{3}}|\Omega|^{q}|y|^{\eta} \psi_{\rho} d y d s \leq \int_{0}^{S_{0}} \int_{\mathbb{R}^{3}}|\Omega|^{q}(1+|y|)^{\eta} d y d s<\infty .
$$

Therefore, we can apply the dominated convergence theorem in the following.

$$
\begin{aligned}
\left|I_{3}\right| & \leq \frac{\|\nabla \sigma\|_{L^{\infty}}}{R(\alpha+1)} \int_{0}^{S_{0}} \int_{\{R \leq|y| \leq 2 R\}}|\Omega|^{q}|y|^{\eta} \psi_{\rho}|y| d y d s \\
& \leq \frac{2\|\nabla \sigma\|_{L^{\infty}}}{(\alpha+1)} \int_{0}^{S_{0}} \int_{\{R \leq|y| \leq 2 R\}}|\Omega|^{q}|y|^{\eta} \psi_{\rho} d y d s \\
& \rightarrow 0
\end{aligned}
$$


as $R \rightarrow \infty$, and

$$
\begin{aligned}
\left|I_{6}\right| & \leq \frac{\|\nabla \sigma\|_{L^{\infty}}}{q R} \int_{0}^{S_{0}} \int_{\{R \leq|y| \leq 2 R\}}|\Omega|^{q}|y|^{\eta} \psi_{\rho}|V| d y d s \\
& \leq \frac{\|\nabla \sigma\|_{L^{\infty}}}{q R} \sup _{R \leq|y| \leq 2 R} \sup _{s \in\left[0, S_{0}\right]} \frac{|V(y, s)|}{|y|} \int_{0}^{S_{0}} \int_{\{R \leq|y| \leq 2 R\}}|\Omega|^{q}|y|^{\eta} \psi_{\rho}|y| d y d s \\
& \leq \frac{2\|\nabla \sigma\|_{L^{\infty}}}{q} \sup _{R \leq|y| \leq 2 R} \sup _{s \in\left[0, S_{0}\right]} \frac{|V(y, s)|}{|y|} \int_{0}^{S_{0}} \int_{\{R \leq|y| \leq 2 R\}}|\Omega|^{q}|y|^{\eta} \psi_{\rho} d y d s \\
& \rightarrow 0
\end{aligned}
$$

as $R \rightarrow \infty$. Here we used the following simple observation: Let $0 \neq y_{1} \in \mathbb{R}^{3}$. Then, $V(y, s)=V\left(y_{1}, s\right)+\int_{0}^{1} \frac{d}{d \tau} V\left(\tau y+(1-\tau) y_{1}\right) d \tau=V\left(y_{1}, s\right)+\int_{0}^{1} y \cdot \nabla V\left(\tau y+(1-\tau) y_{1}\right) d \tau$,

and therefore,

$$
\frac{|V(y, s)|}{|y|} \leq \frac{\left|V\left(y_{1}, s\right)\right|}{|y|}+\sup _{0<\tau<1}\left|\nabla V\left(\tau y+(1-\tau) y_{1}, s\right)\right| \rightarrow 0 \quad \text { as } \quad|y| \rightarrow \infty,
$$

which follows from the hypothesis (1.8). Therefore, passing $R \rightarrow \infty$, and applying the dominated convergence theorem to the other terms of (2.19), we find that

$$
\begin{aligned}
0= & \left(1-\frac{3+\eta}{q(\alpha+1)}\right) \int_{0}^{S_{0}} \int_{\mathbb{R}^{3}}|\Omega|^{q}|y|^{\eta} \psi_{\rho} d y d s-\frac{1}{\alpha+1} \int_{0}^{S_{0}} \int_{\mathbb{R}^{3}}|\Omega|^{q}|y|^{\eta}(y \cdot \nabla) \psi_{\rho} d y d s \\
& -\frac{\eta}{q} \int_{0}^{S_{0}} \int_{\mathbb{R}^{3}}|\Omega|^{q} V \cdot \frac{y}{|y|}|y|^{\eta-1} \psi_{\rho} d y d s-\frac{1}{q} \int_{0}^{S_{0}} \int_{\mathbb{R}^{3}}|\Omega|^{q}|y|^{\eta}(V \cdot \nabla) \psi_{\rho} d y d s \\
& \quad-\int_{0}^{S_{0}} \int_{\mathbb{R}^{3}}(\Omega \cdot \nabla) V \cdot \Omega|\Omega|^{q-2}|y|^{\eta} \psi_{\rho} d y d s \\
& :=J_{1}+\cdots+J_{5} .
\end{aligned}
$$

Under our hypothesis we have $J_{1} \leq 0$. Since $\psi_{\rho}(y)$ is radially non-decreasing, we also have $J_{2} \leq 0$, and

$$
J_{2}=-\frac{1}{\alpha+1} \int_{0}^{S_{0}} \int_{\{\rho \leq|y| \leq \rho+\pi\}}|\Omega|^{q}|y|^{\eta+1} \psi_{\rho}^{\prime} d y d s .
$$

We have

$$
\left|J_{3}\right| \leq \frac{|\eta|}{q} \sup _{|y| \geq \rho, s \in\left[0, S_{0}\right]} \frac{|V(y, s)|}{|y|} \int_{0}^{S_{0}} \int_{\mathbb{R}^{3}}|\Omega|^{q}|y|^{\eta} \psi_{\rho} d y d s \leq \frac{1}{4}\left|J_{1}\right|
$$

for sufficiently large $\rho$. We compute

$$
\begin{aligned}
\left|J_{4}\right| & \leq \frac{1}{q} \int_{0}^{S_{0}} \int_{\mathbb{R}^{3}}|\Omega|^{q}|y|^{\eta}|V| \psi_{\rho}^{\prime} d y d s \\
& \leq \frac{1}{q} \sup _{|y| \geq \rho, s \in\left[0, S_{0}\right]} \frac{|V(y, s)|}{|y|} \int_{0}^{S_{0}} \int_{\{\rho \leq|y| \leq \rho+\pi\}}|\Omega|^{q}|y|^{\eta+1} \psi_{\rho}^{\prime} d y d s \\
& \leq \frac{1}{2}\left|J_{2}\right|
\end{aligned}
$$


for sufficiently large $\rho$. For $J_{5}$ we obtain

$$
\begin{aligned}
\left|J_{5}\right| & \leq \int_{0}^{S_{0}} \int_{\mathbb{R}^{3}}|\nabla V||\Omega|^{q}|y|^{\eta} \psi_{\rho} d y d s \\
& \leq \sup _{|y| \geq \rho, s \in\left[0, S_{0}\right]}|\nabla V(y, s)| \int_{0}^{S_{0}} \int_{\mathbb{R}^{3}}|\Omega|^{q}|y|^{\eta} \psi_{\rho} d y d s \\
& \leq \frac{1}{4}\left|J_{1}\right|
\end{aligned}
$$

for sufficiently large $\rho$. Taking into account the estimates (2.25) $-(2.27)$ in (2.23), we find that there exists $\rho_{0}>0$ such that

$$
\begin{aligned}
& 0 \geq \frac{1}{2}\left(\frac{3+\eta}{q(\alpha+1)}-1\right) \int_{0}^{S_{0}} \int_{\mathbb{R}^{3}}|\Omega|^{q}|y|^{\eta} \psi_{\rho} d y d s \\
& \quad+\frac{1}{2(\alpha+1)} \int_{0}^{S_{0}} \int_{\mathbb{R}^{3}}|\Omega|^{q}|y|^{\eta}(y \cdot \nabla) \psi_{\rho} d y d s
\end{aligned}
$$

for all $\rho \geq \rho_{0}$. Hence, $\Omega(y, s)=0$ for all $(y, s) \in\left\{y \in \mathbb{R}^{3}|| y \mid>\rho_{0}\right\} \times\left[0, S_{0}\right]$. Applying Theorem 1.3 (i)(a), we conclude $\Omega=0$.

\section{Acknowledgements}

The author would like to thank to the anonymous referees for useful suggestions. This research is supported partially by NRF Grant 2006-0093854 and 2009-0083521.

\section{References}

[1] J. T. Beale, T. Kato and A. Majda, Remarks on the breakdown of smooth solutions for the 3-D Euler equations, Comm. Math. Phys., 94, (1984), pp. 61-66.

[2] D. Chae, Nonexistence of asymptotically self-similar singularities in the Euler and the NavierStokes equations, Math. Ann., 338, (2007), pp. 435-449.

[3] D. Chae, K. Kang and J. Lee, Notes on the asymptotically self-similar singularities in the Euler and the Navier-stokes equations, Discrete Contin. Dyn. Syst. , 25, no. 4, (2009), pp. 1181-1193.

[4] D. Chae and R. Shyvdkoy On formation of a locally self-similar collapse in the incompressible Euler equations, Arch. Rational Mech. Anal., 209, no. 3, (2013), pp. 999-1017.

[5] D. Chae and T-P. Tsai, On discretely self-similar solutions of the Euler equations, Math. Res. Lett., 21, no.3 (2014), pp. 1-11.

[6] P. Constantin, C. Fefferman and A. Majda, Geometric constraints on potential singularity formulation in the 3-D Euler equations, Comm. P.D.E., 21, (3-4), (1996), pp. 559-571. 
[7] J. Eggers and M. A. Fontelos, The role of self-similarity in singularities of partial differential equations, Nonlinearity, 22, (2009), pp. 1-44.

[8] L. Escauriaza, G. Seregin and V. Šverák , $L_{3, \infty}$-Solution to the Navier-Stokes Equations and Backward Uniqueness, Russ. Math. Survey, 58, no. 2, (2003), pp. 211250 .

[9] Y. Giga and R.V. Kohn, Asymptotically self-similar blow-up of semilinear heat equations, Comm. Pure Appl. Math., 38, (1985), pp. 297-319.

[10] Y. Giga and R.V. Kohn, Characterizing blow-up using similarity variables, Indiana Univ. Math. J., 36, (1987), pp. 1-40.

[11] S. Gustafson, K. Kang and T-P. Tsai, Regularity criteria for suitable weak solutions of the Navier-Stokes equations near the boundary, J. Diff. Eqns, 226, no. 2, (2006), pp. 594-618.

[12] T. Hou and R. Li, Nonexistence of locally self-similar blow-up for the 3D incompressible Navier-Stokes equations, Discrete Contin. Dyn. Syst. , 18, no. 4, (2007), pp. 637-642. Math. Z., 187, (1984), pp. 471-480.

[13] A. Majda and A. Bertozzi, Vorticity and Incompressible Flow, Cambridge Univ. Press. (2002).

[14] J. R. Miller, M. O'Leary and M. Schonbeck, Nonexistence of singular pseudoself-similar solutions of the Navier-Stokes system, Math. Ann., 319, no. 4, (2001), pp. 809-815.

[15] J. Nečas, M. Ružička and V. Šverák, On Leray's self-similar solution of the Navier-stokes equations, Acta Math., 176, no. 2, (1996), pp. 283-294.

[16] R. Pelz, Symmetry and hydrodynamic blow-up problem, J. Fluid Mech., 444, pp. 299-320.

[17] M. Schonbek, Nonexistence of pseudo-self-similar solutions to incompressible Euler equations, Acta Math. Sci. Ser. B, Engl. Ed. 31, no. 6, (2011), pp. $2305 ? 2312$.

[18] G. Seregin, A certain necessary condition of potential blow-up for Navier-Stokes equations, Comm. Math. Phys., 312, no.3, (2012), pp. 833-845.

[19] T-P. Tsai, Forward Discrete Self-Similar Solutions of the Navier-Stokes equation, Comm. Math. Phys., 328, no. 1, (2014), pp. 29-44.

[20] T-P. Tsai, On Leray's self-similar solution of the Navier-stokes equations satisfying local energy estimates, Arch. Rational Mech. Anal., 143, no. 1, (1998), pp. 29-51. 